\title{
Systems biology - old concepts, new science, new challenges
}

\section{Raina Robeva*}

Department of Mathematical Sciences, Sweet Briar College, Sweet Briar, VA, USA

*Correspondence: robeva@sbc.edu

Life is a relationship among molecules and not a property of any molecule.

Linus Pauling

On the timescale of history of science, Systems Biology is so new that a precise consensus definition for the term is yet to be developed. However, the common-sense concept behind systems biology that a living organism is more than a mechanical sum of its parts has been noted, discussed, and analyzed for centuries by philosophers and scientists alike, with Kant, Goethe, Schrödinger, and Delbruck being just a few examples. It is now well understood that life at any level - from cell functioning to human behavior - is defined by the dynamical interactions between its components, not by the properties of these components in isolation. However, only recently fundamental advances in molecular biology, computing, and mathematical modeling, have allowed us to attempt a coordinated effort to studying and understanding this phenomenon.

Systems biology is the cross-disciplinary methodology behind the effort to understand the dynamics of life, aiming at determining how the individual components of a living system interact in space and time to form functional networks. The challenges are huge: in biological systems at all levels of organization from sub-cellular to the cell, tissue, organ, and human behavior, control and functional mechanisms are emergent properties of networks, not of their separate components. These biological networks exhibit self-organization with relatively independently functioning sub-systems that have network structures of their own, often comprised of modular units found in multiple locations. A number of questions of equal importance arise in this context. To name a few (reflecting the author's personal bias and interest): What network interactions lead to a specific system function? Knowing a system's function, can a network of subcomponents be identified to exhibit the same behavior? In what way are self-organizing biological networks between organizational levels and between organisms similar? In what way are they different?

The tools of modern biology and classical neuroscience are insufficient in seeking answers. Their insights into available data from molecular biology, genomic, proteomic, metabolomic, neuroendocrine, and behavioral research need to be merged with mathematical models, computational tools, and engineering systems analysis to ensure better understanding of evolution, physiology and human behavior. In this effort, tight links between network modeling and experimentation and their consistent iterative interaction are critical to understanding the network structure. In other words, the network structure and interactions that are inherent within biological systems need to be deciphered using modeling, simulation, and system analysis.

However, there are no routine methods for doing that and the process is far from straightforward. For instance, it may be experimentally unclear which biomolecular variables to measure due to the fact that multiple feedback loops are present to control the mechanisms of molecular interactions. The problems may be alleviated if we could compartmentalize the experimental and analytical challenges into experimentally observable (and thus more tractable) problems:

First, at the level of biology, a complex system can be theoretically decomposed into relatively independent and simpler modules (sub-networks) that act as regulatory elements. Then, targeted experiments can be performed to elucidate the function of each sub-system and the between-module interactions. This modular approach is also mathematically appealing because, from a mathematical viewpoint, some modules can be considered as functions whose inputs are determined by the rest of the network elements but are otherwise largely independent from the links between them. The approach can be utilized to build synthetic bionetworks that exhibit desired predetermined properties. The work by Elowitz and Leibler (2000) of the construction of an artificial genetic regulatory network known as the repressilator, is a well-known example leading to an oscillating circuit. Developing methodologies for identifying the structure of complex networks in terms of their simpler functional components, together with identifying system responses predicated on the network topology, are of utmost importance and represent areas of active research.

Second, at the level of modeling, converting biological network maps into dynamical models is equally important and challenging. Three major types of approaches could be mentioned here, generally classifying the dynamic modeling tools into three [overlapping] classes: (i) models to understand system structure, functionality and network interactions; (ii) models to simulate system evolution and dynamics, and (iii) models to control systems' design properties or realtime behavior. Historically, most developed is the class of models used to understand system properties. Such models are typically parsimonious, including a minimal number of key functional elements and interactions that describe the structure or the principal dynamics of the system. When the knowledge about a specific biosystem evolves and details about its elements emerge, the second type of models makes feasible the computer simulation of system behavior. Simulation models are typically as comprehensive as possible, including as many system elements and interactions as possible in an attempt to approximate in silico the system dynamics observed in vivo. Finally, a detailed understanding of system functionality would allow the construction of the third type models (and presumably devices) that are capable of controlling system properties by setting initial design conditions, or by real-time feedback control.

Meeting the challenge of understanding, simulating, and controlling the dynamic and the structural properties of biological 
networks has a significant potential toward developing new methods for predictive and preventive medicine. From reverse-engineering of cellular networks (Stolovitzky et al., 2007), through advances in modeling and simulations of the human metabolism and hopes for building an artificial pancreas for the management of diabetes (Cobelli et al., 2009), to new methods for confronting drug and alcohol addition based on the understanding of the neural circuits regulating human behavior (Johnson, 2004), the systems approach provides a unifying platform for research breakthroughs. Significant advances have already been made, and the future of systems biology undoubtedly holds even greater promises for overcoming the challenges of further understanding biological complexity.

\section{REFERENCES}

Cobelli, C., Dalla Man, C., Sparacino, G., Magni, L., Nicolao, G., and Kovatchev, B. P. (2009). Diabetes: models, signals, and control. IEEE Rev. Biomed. Eng. 2, 54-96.

Elowitz, M. B., and Leibler, S. (2000). A synthetic oscillatory network of transcriptional regulators. Nature 403, 335-338.

Johnson, B. A. (2004). Progress in the development of topiramate for treating alcohol dependence: from a hypothesis to a proof-of-concept study. Alcohol. Clin. Exp. Res. 28, 1137-1144.
Stolovitzky, G., Monroe, D., and Califano, A. (2007). Dialogue on reverse-engineering assessment and methods: the DREAM of high-throughput pathway inference. Ann. N. Y. Acad. Sci. 1115, 1-22.

Received: 19 August 2009; accepted: 04 January 2010; published online: 18 January 2010.

Citation: Robeva R (2010) Systems biology-old concepts, new science, new challenges. Front. Psychiatry 1:1. doi: 10.3389/fpsyt.2010.00001

This article was submitted to Frontiers in Systems Biology a specialty of Frontiers in Psychiatry.

Copyright (c) 2010 Robeva. This is an open-access article subject to an exclusive license agreement between the authors and the Frontiers Research Foundation, which permits unrestricted use, distribution, and reproduction in any medium, provided the original authors and source are credited. 